\title{
Space-Charge Compensation in Proton Boosters
}

\author{
A.V. Burov, G.W. Foster, V.D. Shiltsev \\ FNAL, P.O. Box 500, Batavia, IL 60510
}

\begin{abstract}
Recently, it was proposed to use negatively charged electron beams for compensation of beam-beam effects due to protons in the Tevatron collider [1]. We show that a similar compensation is possible in space-charge dominated low energy proton beams. The idea has a potential of several-fold increase of the FNAL Booster beam brightness. Best results will be obtained using three electron lenses around the machine circumference, using co-moving electron beam with time structure and profile approximately matched to the proton beam. This technique, if feasible, will be more cost effective than the straightforward alternative of increasing the energy of the injection linac.
\end{abstract}

\section{INTRODUCTION}

Defocusing repulsive forces due to self space charge lead to degradation of high-intensity particle beams. In circular accelerators, the figure of merit of such an effect is shift of incoherent betatron oscillations [2]:

$$
\Delta \nu_{S C}=-\frac{N_{t o t} r_{c} B_{f}}{4 \pi \varepsilon_{n} \beta_{p} \gamma_{p}^{2}}
$$

where $N_{t o t}$ is total number of particles in the ring, $r_{c}=$ $1.53 \cdot 10^{-18} \mathrm{~m}$ for protons, $\varepsilon_{n}$ is rms normalized emittance, $\beta_{p}=v_{p} / c$ and $\gamma_{p}$ are usual relativistic parameters, and $B_{f} \geq 1$ is a peak to average current ratio. Normally, for proton low-energy synchrotrons the tune shift lays in range of $-0.1 \ldots-0.5$ (see, e.g.,[4]). Above the threshold, the beam emittance dilute and particles are lost. Due to the acceleration, the short time at low energy is enough for developing only the lowest order resonances.

Compensation of space charge forces on average, can significantly improve dynamics in low-energy accelerators, and allow higher beam intensities.

\section{COMPENSATION OF SPACE CHARGE FORCES BY ELECTRON BEAMS}

Negatively charged electron beams can be used for compensation of space-charge effects in low energy proton beams the same way as in the ongoing Tevatron Electron Lens (TEL) project to compensate beam-beam effects due to protons in the Tevatron collider [1]. Protons going through the electron beam experience focusing force which has opposite sign to self space charge force and can precisely fully or partially compensate the latter.

Practically, the electron beam set-up could occupy only a small fraction of the ring circumference $C$. Therefore, the kick experienced by every proton could be compensated only in average. In the same time, the proton bunch structure can be matched by fast modulation of the electron current.

Assuming $\kappa$ being a degree of the compensation, the tune shift on the electron beam can be presented as

$$
\Delta \nu_{e} \equiv \frac{\beta_{r}}{4 \pi} \frac{\left(1-\beta_{e} \beta_{p}\right) J_{e} L r_{c}}{e \beta_{e} c \sigma_{e}^{2} \gamma_{p} \beta_{p}}=-\kappa \Delta \nu_{S C},
$$

where $J_{e}$ is electron current, $\sigma_{e}$ is the rms electron beam radius, $L$ is interaction length, $\beta_{r}$ is beta function at the location of the electron lens.

The necessity of fast longitudinal electron current modulation for matching the proton bunch profile requires for electrons to move in the same direction as protons.

Assuming the rms size of the electron beam matching the proton beam size in the location of the electron lens $\sigma_{e}^{2}=$ $\varepsilon_{n} \beta_{p} \gamma_{p} \beta_{r}$, then we obtain the necessary electron current in the lens:

$$
J_{e}=\kappa J_{p} B_{f} \frac{C}{L} \frac{\beta_{e}}{\gamma_{p}^{2} \beta_{p}^{2}\left(1-\beta_{e} \beta_{p}\right)},
$$

As it is shown in the next Section, optimum compensation requires $\kappa \simeq 0.3$.

\section{COHERENT MODES AND OPTIMAL COMPENSATION}

The beam own space charge forces shift down both single-particle (incoherent) modes and the beam coherent modes. An exception is the coherent dipole mode (beam centroid motion) which does not depend on the space charge, when the image charges are neglected. If one of these modes is in resonance with the focusing lattice (structure resonance), the mode becomes unstable, that leads to the beam loss and/or growth of the beam emittance. An important point is that the space charge and electron tune shifts are mode-specific, so if the electron lens compensates the space charge tune shift for one of the modes, it does not compensate it for others. Therefore, the electron lens can increase the proton brightness threshold but can not eliminate it. The incoherent tune shift introduced by the electron lens $\Delta \nu_{e}$ can be considered as proportional to the Laslett tune shift $\Delta \nu_{S C}$, i. e. $\Delta \nu_{e}=-\kappa \Delta \nu_{S C}$, with compensation coefficient $\kappa$ to optimize.

In the smooth approximation, assuming approximately equal vertical and horizontal lattice tunes $\nu_{x} \approx \nu_{y} \approx \nu_{0}$, and small tune shifts $\Delta \nu<<\nu_{0}$, the single-particle frequency goes as

$$
\nu=\nu_{0}+(\kappa-1) \Delta \nu_{S C} .
$$


In this case, the coherent tunes of the dipole and two envelope modes (subscripts "d", "," and "-") are given as

$$
\begin{aligned}
& \nu_{d}=\nu_{0}+\kappa \Delta \nu_{S C} \\
& \nu_{+}=2 \nu_{0}+(2 \kappa-1) \Delta \nu_{S C} \\
& \nu_{-}=2 \nu_{0}+(2 \kappa-3 / 2) \Delta \nu_{S C} .
\end{aligned}
$$

A consequence of these simplified expressions is that an exact compensation of the space charge tune shift for all the three tunes, or even for any two of them, is impossible; if one of the modes is compensated, the two others are not. Instead, there is a best compensation degree $\kappa$, with $\Delta \nu_{0}=\kappa \Delta \nu$, where the stopping resonance is reached at the maximal space charge.

Table 3 summarizes data from various space-charge limited low-energy proton synchrotrons [4] - the first row shows bare lattice tunes $\nu_{x}$ and $\nu_{y}$, the second row contains maximum empirically achieved Laslett tune shift for each machine $\Delta \nu$ exp. We compare experimental values with calculated distance $\Delta \nu_{\text {inc }}$ to the nearest half-integer resonances: incoherent (third row) and coherent $\Delta \nu_{\text {coh }}$ (bottom row), calculated as in Ref. [3].

The table reveals some noticeable features. First, the empirically reached Laslett tune shifts significantly exceed the single-particle limits $\Delta \nu_{\text {inc. }}$. Second, for all machines but AGS and AGS-Booster $\Delta \nu_{\exp }$ is close to the threshold of the envelope instability $\Delta \nu_{\mathrm{coh}}$. This leads to a conclusion that the incoherent resonances may not be important; the actual threshold is rather determined by the coherent instabilities. This conclusion agrees with the analysis of Ref.[3]. Finally, the resonances at $\Delta \nu_{\mathrm{coh}} \leq 0.1$ do not reveal themselves, perhaps, due to a weak space charge at the resonance crossing.

Behavior of the modes changes when the compensation is applied. Dynamics of the dipole mode is rather different from the envelope modes, that makes it difficult to even double the threshold by varying the compensation degree $\kappa$, because one or another mode should cross linear resonance. Threshold Laslett tune shift of about 0.6 can be achieved with $\kappa=0.33$ (a factor of $\approx 1.6$ in comparison with the no-compensation case).

An additional option is associated with a possibility to have the degree of compensation $\kappa$ variable and dependent on the Laslett tune shift. In that case, modulation of the electron beam current is almost as easy as for the constant $\kappa$ strategy. The variable rate allows to jump over the resonances, which could additionally increase the space charge limits.

Symmetry of an accelerator focusing lattice is important for the space-charge compensation. If the lattice consists of $P$ identical periods, then strong and wide structure linear resonances occur at coherent/incoherent frequencies $\nu_{\text {coh, inc }}=P m / 2$, where $m$ is integer.

Thus, more than one compensation set-up is needed to keep nearest linear resonances as non-structure. For example, the Fermilab Booster has periodicity $P=24$, and tunes $\nu_{x, y}=(6.7,6.8)$. With two electron lenses symmetrically placed in opposite parts of the ring, the effective periodicity is reduced to $P=2$. Thus, the nearest incoherent resonance $\nu=6.5$ is non-structure (as it is without compensation devices at $P=24$ ), but the envelope resonances at $\nu_{-,+}=13=P m / 2=2 \cdot 13 / 2$ become structure. As the result, three identical compensators symmetrically positioned at the orbit are required in order to preserve nonstructure weakness of the nearest incoherent and coherent resonances.

\section{NUMERICAL EXAMPLE: FERMILAB BOOSTER}

Let us consider the space-charge compensation in the Fermilab Booster. Parameters of FNAL Booster are presented elsewhere $[5,4])$. The space charge tune shift at injection is about

$\Delta \nu_{S C}(t)=-0.435 \cdot B_{f}(t) \cdot\left(\left[\varepsilon_{n} \beta_{p} \gamma_{p}^{2}\right](t) /\left[\varepsilon_{n} \beta_{p} \gamma_{p}^{2}\right](t=0)\right)$

if parameters are $N_{t o t}=5 \cdot 10^{12}, \varepsilon_{n}=1 \pi \mathrm{mm} \cdot \mathrm{mrad}$, $\gamma_{p}=1.4, \beta_{p}=0.7$. The period of strong space-charge action is comparatively short - injection itself takes about 12 turns, then some 20-40 turns the beam circulates without RF to wipe off the Linac RF structure - it corresponds to $B_{f}=1$. After that the Booster RF is being adiabatically turned on over some 100 turns, and then the beam energy rapidly grows, e.g., to $2.5 \mathrm{GeV}$ after about 3000 turns. Note, that the ratio of peak to average current rapidly increases from 1 to $B_{f}(t) \approx 2-3$ over a hundred turns, while the factor $1 /\left(\beta_{p} \gamma_{p}^{2}\right)$ slowly decreases 4 times from 0.71 to 0.174 over thousands of turns. If there would be no particle loss and emittance blow up then the Booster beam space charge tune shift parameter would reach the maximum value of right after bunching $\Delta \nu_{S C} \approx 0.9-1.3$.

Currently, due to space charge effects, the beam loses some $35-40 \%$ of particles during that initial period of 5$6 \mathrm{~ms}$, and its transverse emittance blows up about 3 times [6]. From that we can conclude that the beam brightness deteriorates quickly if the space charge tune shift exceeds $\Delta \nu_{S C} \geq-(0.25-0.4)$, that is consistent with conclusions of previous studies in the Booster [7].

According to our analysis in the previous Section, the optimal compensation coefficient is less than $1, \kappa=0.33-$ 0.55 , therefore, we can present the required peak electron current as

$$
J_{e}=\left(B_{f} \cdot \kappa\right) \cdot \frac{e c N_{t o t}}{L N_{l}} \cdot \frac{\beta_{e}}{\gamma_{p}^{2}\left(1-\beta_{p} \beta_{e}\right)} .
$$

As concluded in Section 3 above, it is beneficial to install $N_{l}=3$ equally strong lenses in the Booster, so if each is $L=4 \mathrm{~m}$ long (to fit $6 \mathrm{~m}$ long drift sections), then for $B_{f}=3$ and $N_{t o t}=5 \cdot 10^{12}$ we yield $J_{e}(\kappa=0.33)=8.3 \mathrm{~A}$ of the peak current in each of three $70 \mathrm{kV}$ electron beams, $\beta_{e}=0.52$. The choice of the electron beam energy and $\beta_{e}$ is made to satisfy condition $\sigma_{s} \approx L\left(\beta_{p}-\beta_{e}\right) / \beta_{p}$, where the rms proton bunch length of $\sigma_{s}=1 \mathrm{~m}$ is taken. If the 


\begin{tabular}{|l|c|c|c|c|c|r|r|}
\hline Parameter & KEK-B & FNAL-B & ISIS & AGS & AGS-B & CERN PS & CERN PS-2 \\
\hline$\nu_{x} / \nu_{y}$ & $2.17 / 2.3$ & $6.7 / 6.8$ & $3.7 / 4.2$ & $8.75 / 8.75$ & $4.8 / 4.9$ & $6.22 / 6.22$ & $6.22 / 6.28$ \\
\hline$\Delta \nu_{\exp }$ & 0.23 & 0.4 & 0.4 & 0.58 & 0.5 & 0.27 & 0.36 \\
\hline$\Delta \nu_{\text {inc }}$ & 0.17 & 0.2 & 0.2 & 0.25 & 0.3 & 0.22 & 0.22 \\
\hline$\Delta \nu_{\text {coh }}$ & $0.27 / 0.08$ & $0.36 / 0.08$ & 0.32 & 0.33 & $0.07 / 0.2$ & 0.27 & 0.33 \\
\hline
\end{tabular}

Table 1: Laslett tune shift reached empirically $\Delta \nu \exp$ at various low-energy synchrotrons as compared with calculated Laslett tune shifts related to the nearest incoherent $\Delta \nu_{\text {inc }}$ and coherent $\Delta \nu_{\text {coh }}$ resonances.

ultimate goal is to double the Booster intensity - that is allowed by the FNAL Linac and will require more Booster RF power - then $J_{e}(\kappa=0.33)=16.6$ A of the electron current are needed.

In general, the Booster Electron Lens (BEL) will be much alike the Tevatron Electron Lens (TEL) for compensation of beam-beam effects [1].

The BEL will consist of solenoid magnets for electron beam transport, electron gun and collector, high voltage modulator, vacuum system, beam diagnostics and control. The $4 \mathrm{~m}$ long interaction region has to be inside a pair of 2-m long 2-4 kG solenoid magnets with opposite polarity of the magnetic field. Such a configuration will provide zero field integral $\int B d z=0$ and, thus, will minimize the Booster focusing lattice perturbation. Magnetic field lines in these magnets have to be straight within $1 \mathrm{~mm}$ (some $20 \%$ of the proton beam size); a number of additional dipole corrector coils are needed for the electron beam steering. Shorter 2-4 kG side solenoids are necessary for electron beam injection and extraction. It may be necessary to add two toroids to make the 90 degrees turns smoother. This will be the subject of more detailed studies. A lot of subsystems, including the gun and collector, beam diagnostics, HV pulser can be just copied and/or upgraded from the Tevatron Electron Lens set-up, see Ref.[1] and references therein.

\section{SUMMARY}

The use of low-energy high-current electron lenses to compensate space charge effects in high-brightness proton accelerators looks very promising. Our consideration shows that in optimal configuration few (three) electron lenses can compensate space charge tune shifts as large as $\Delta \nu_{S C} \approx 0.6-1.2$. With further increase of the proton beam brightness some coherent modes or incoherent motion can become unstable while crossing particular resonances.

We have considered electron lenses for the compensation in the Fermilab Booster. We found that electron beam systems are quite feasible for realization of either proton beam emittance upgrade or two-fold intensity upgrade in the Booster. These upgrades can significantly improve the performance of fixed target experiments, e.g., NuMI; and/or will allow to increase the antiproton production, proton beam brightness in collision and ultimately increase the luminosity of the Tevatron.

In principle, there are other tools to compensate the tune shift but they all have serious disadvantages. For example, pulsed $\gamma_{t}$-jump quadrupoles in the Booster can shift the tune for about 2, but it does not seem possible to modulate their gradients over the bunch length of few ns. RF quadrupoles can provide necessary modulation along the bunch but will require very powerful RF sources.

The proposed electron lens has a number of advantages: easier beam shape control and current modulation, moderate power consumption. The parameters of the lens are quite feasible that make the proposal attractive for further consideration and practical implementation.

The proposed compensation can also allow a substantial brightness increase in low energy proton and ion storage rings with electron cooling, as many of them are spacecharge dominated.

Space charge compensation in low energy $(\sim 1 \mathrm{MeV})$ coasting proton beams by ionization electrons was reported in [8]. The compensation allowed many-fold increase of the proton intensity compared to the proton space-charge limit. We think that advantages of the well-controlled electron beams will allow to achieve comparable success in highenergy bunched proton beams as well.

We acknowledge helpful discussions with R.Webber, J.Lackey, R. Baartman, J.Marriner, S.Y.Lee, V.Dudnikov and S.Holmes.

\section{REFERENCES}

[1] V. Shiltsev, et.al, this conference; see also Phys.Rev.ST-AB, 2, 071001 (1999).

[2] M. Reiser, Theory and Design of Charged Particles Beams, John Wiley\&Sons (1994).

[3] R. Baartman, KEK Proceedings 95-7 (1995), 273.

[4] W. Chou, in ICFA Beam Dynamics Newsletter, No.20 (1999).

[5] E.L. Habbard, FNAL TM-405 (1973).

[6] R. Webber, FNAL-Conf-00/193 (2000).

[7] C. Ankenbrandt, S. Holmes, in Proc. 1987 IEEE PAC, Washington, D.C., v.2, (1987), 1066

S. Holmes, in AIP Conf. Proc. 377 (1995), 42.

[8] Yu. Belchenko, et.al, in Proc. IX Int. Part. Accel. Conf., v.2, Protvino (1977), 287. 